\title{
A Non-linear Programming Model for the Machine Grouping and a Genetic Algorithm based Solution Methodology
}

\author{
C.R Shiyas \\ National Institute of Technology Calicut, \\ Calicut, Kerala, India, 673601
}

\author{
V. Madhusudanan Pillai \\ National Institute of Technology Calicut, \\ Calicut, Kerala, India, 673601
}

\begin{abstract}
Cellular Manufacturing is an important application of group technology principles and is suitable in a medium variety medium volume production environment. It is concerned with the production of part types in a flow line manner by dividing the production system into manufacturing cells. In cellular manufacturing system (CMS) design, cell formation is one of the most important steps which contain identification of machine cells and part families. Usually, minimization of intercell movements is the criteria for CMS design. In this paper, we introduce a heterogeneity concept which indicates diversity of machines in a cell and it is measured based on the machine assigned to a cell and machines required for processing of parts visiting the cell. A non-linear integer programming model for the design of manufacturing cells is proposed in this paper to minimize the heterogeneity of cells formed for the given part-machine incidence matrix. The solution is found through a heuristic procedure based on a genetic algorithm coded in MATLAB. The approach produced solution with a grouping efficacy equal to or better than some of the previous approaches based on seven problems.
\end{abstract}

\section{General Terms}

Cellular manufacturing system, mathematical model, Genetic algorithm based solution methodology.

\section{Keywords}

Cellular manufacturing systems, Heterogeneity of cells, Partmachine incidence matrix, Genetic algorithm

\section{INTRODUCTION}

Manufacturing industry is under intense pressure due to the present competitive market situations and achieving maximum productivity is always in the forefront of such companies' objectives. Managers across the world are striving to adopt new manufacturing practices to attain this goal. Cellular manufacturing $(\mathrm{CM})$ is a manufacturing practice that uses group technology philosophy to achieve a flow-line production through efficient configuration of facilities. CM is an approach that helps to manufacture a variety of products with as little waste as possible. Factories changed to cellular manufacturing are benefited by the reduction of overproduction and waste, shorter lead time, less work in process inventories, improved quality and productivity, improved teamwork and communication among cell operators, etc.

The group technology (GT) orientation in manufacturing was first presented by Flanders in 1925. In 1959 Mitrofonov published a book on scientific principles of GT and Burbidge in 1960 proposed a systematic planning approach for GT called production flow analysis. From then onwards there has been several methods, models and algorithms developed for finding the solution for the fundamental problem of CMS design of forming part families and machine cells. The objective of a general manufacturing cell design problem is to obtain a satisfactory grouping of parts into families and machines into cells for a given a set of parts and available resources with different operations requirements. Majority of the CMS design methods use part-machine incidence matrix as the input for forming part families and machine cells and the aim is to get an output matrix with a good block diagonal structure [1], [2]. Many models were also developed in this area considering attributes like sequence of operations [3], dynamic demand and machine capacity $[4,5]$ alternate routes, setup cost, tool consumption cost, etc. [6], machine failures [7].

In general, the literature considers the minimisation of intercell movements while designing a CMS. But, this paper introduces a concept called heterogeneity as a criterion for designing a CMS. Heterogeneity is a measure used in the proposed model which is depending on the machines assigned to a cell and the machines required for processing of parts visiting the cell. Every part visiting a cell contributes to the heterogeneity of the cell. Heterogeneity due to a part visiting a cell is the number of machines present in a cell which are not required for the part to process. Heterogeneity of a cell is the sum of the heterogeneity of parts visiting the cell. Heterogeneity of CMS is the sum of the heterogeneity of all cells. This paper proposes a non-linear mathematical model which considers the minimisation of heterogeneity for a given number of cells. Generally, number of cells is a parameter fixed by the management for the design of CMS. The partmachine incidence matrix is the basic input for the model of this paper. A genetic algorithm based solution methodology is developed for the proposed model.

\section{REVIEW ON EARLY METHODS}

Array-based clustering methods are the simplest category of manufacturing cell design methods. The array based techniques try to assign machines to different cells and parts into families by rearranging the order of rows and columns of the part machine incident matrix to find a block diagonal form. Examples of array-based clustering methods include the bond energy algorithm (BEA) of McCormick [8], rank order clustering (ROC) of King [1], etc and these methods make use of a series of column and row permutations to form part families and machine cells simultaneously. Hierarchical clustering methods operate on an input data set based on some similarity or distance measures (generally termed as similarity 
coefficients) and produce a hierarchy of clusters or partitions. McAuley [9] used the single linkage clustering (SLC) and Seifoddini [10] applied the average linkage clustering (ALC) algorithm to identify machine groups from the distance matrix. These methods have the limitation of not forming part families and machine cells simultaneously. Examples of nonhierarchical methods include GRAFICS, of Srinivasan and Narendran [11], ZODIAC, of Chandrasekharan and Rajagopalan [12], etc. Non-hierarchical methods are generally found to be better than the previous methods; based on a comparative analysis of efficiency measures like grouping efficiency and grouping efficacy. Graph partitioning approaches are employed in CMS design by treating the machines and/or parts as nodes and the processing of parts as arcs connecting the nodes [13]. Objective of these models is to obtain disconnected sub-graphs from a machine-machine or machine-part graph to identify manufacturing cells. These methods are found to be depending on the initial pivot element choice.

\section{REVIEW OF MATHEMATICAL MODELS AND ALGORITHMS IN CMS DESIGN}

The CMS design problems are combinatorial optimization problems and mathematical programming approaches are very much suitable for getting good solutions. Mathematical models generally treat the manufacturing cell formation problem as a mathematical optimization problem. Numerous models have been developed by various researchers based on mathematical programming and the main advantage of mathematical models is the easiness of incorporation of different attributes to the model by the designer. Choobineh [14] developed a model based on integer programming, where it first determines machine cells and then assigns part families to cells with an objective of minimizing the costs. Srinivasan et al. [15] proposed an assignment model which linked the principles of assignment problem of operations research into cell design. But, the main drawback was that the part grouping was done before assigning it to a cell. Adil et al. [16] developed an assignment allocation algorithm (AAA) for the identification of part families and machine groups simultaneously. But their model may lead to formation of cells without any machines, but with part assignment. A mathematical model is developed by Yashuda et al. [17] using multi-objective decision making for CMS design and will direct to a variety of solutions.

Identifying a solution methodology to the mathematical model is another important decision that has to be made. Many models incorporate a wide variety of system attributes in the model and hence the model may be complicated. So, solving it using traditional methods will be difficult. Using software packages for solving CMS design models usually takes very high time for computation and hence it will be difficult to deal with large problems. In such situations, nontraditional optimization techniques like genetic algorithms [5], tabu search[18], simulated annealing [19] are often applied as solution methodology. Heuristic-based algorithms [2] are also seen in CMS design literature because sometimes it will be difficult to model the entire situation in a mathematical form due to the complexity of the system.

Previous researchers differ in various types of attributes used. However, almost all are using minimization of intercell movements as one of the criteria for the design of CMS. In this paper we suggest the minimization of heterogeneity of CMS as the objective function. Block diagonalisation of the part-machine incidence is the initial approach in CMS design. This paper also proposes the cell design in block diagonal form using the heterogeneity as the objective. This heterogeneity is similar to the concept of heterogeneity of operations in a cell used by Diaz and Lozano [18] for the design of dedicated cells. Genetic algorithm is used as a solution procedure and this method is applied to the problems of the literature. The solutions are compared using grouping efficacy. The results of the proposed method are found to give better solution.

\section{PROPOSED MATHEMATICAL MODEL}

The proposed non linear integer programming model has the objective function as the minimization of heterogeneity of cells formed for the given machine-part incidence matrix. The number of cells is a managerial input to this model. The model assigns machines to given cells by minimizing the heterogeneity. Notations used are given below.

$$
\begin{aligned}
& i \quad-\text { Index of machines, } i=1,2, \ldots, M \text {. } \\
& k \quad-\text { Index of cells } 1,2, \ldots, \mathrm{C} \text {. } \\
& l \quad-\text { Index of parts } \\
& v_{i l}=1 \text { if part type } l \text { requires operation in machine of }
\end{aligned}
$$

$\operatorname{Min} Z=\sum_{l} P_{l}$

Subject to

$$
\sum_{k=1}^{C} x_{i k}=1 \quad \forall i
$$

$$
\begin{aligned}
& \sum_{i=1}^{M} x_{i k} \geq 1 \quad \forall k \\
& \sum_{i=1}^{M} x_{i k} v_{i l} \leq M S_{l k} \quad \forall i, k \\
& P_{l}=\sum_{k=1}^{C} S_{l k} \sum_{i=1}^{M} x_{i k}\left(1-v_{i l}\right) \quad \forall l \\
& x_{i k,} S_{i, k} \in\{0,1\}
\end{aligned}
$$


The following discussion gives the description of the above equations/inequality.

(1) - The objective function is the minimisation heterogeneity.

(2) - Ensures the presence of one machine type in one cell alone.

(3) - Ensures at least one machine in each cell

(4) - Defines $S_{i k}$ which is used for calculating heterogeneity

(5) - Calculate $P_{l}$ (Heterogeneity)

(6) - Binary constraints

\section{GENETIC ALGORITHM BASED SOLUTION METHODOLOGY}

The solution methodology involves development of genetic algorithm for the given cell configuration, part assignment, performance valuation of the grouping using grouping efficacy and selection of the best part family/cell formation based on grouping efficacy.

\subsection{Genetic Algorithm}

A genetic algorithm (GA) based solution procedure is used for the above formulation. Genetic algorithms are search techniques for global optimisation in a complex search space. They combine the survival of the fittest among solution structures with structured, randomized search strategy in which new strong solutions displace weak solutions in successive iterations [20]. It is an intelligent exploitation of a random search within a defined search space to solve a problem [21].

Genetic algorithm usually starts with an initial set of solutions called population and the population at a given time is called a generation. Each individual in the population is called a chromosome.

\subsubsection{Chromosome representation}

For the cell formation problem discussed here, each gene represents a cell number and the position of the gene in the chromosome represents the machine number. The length of the chromosome represents the number of machine types considered. For example, a string ' 312311 ' represents a three cell solution with machines in each cell are as follows:

Cell 1: machines 2, 5, 6

Cell 2: machine 3

Cell 3: machines 1,4

\subsubsection{Initialisation of Population}

The initialisation process is carried out with a randomly generated solution. The initial solution is generated only once at the beginning and is called old population in the first generation of the genetic algorithm. The population size remains constant from generation to generation and has considerable effect on the performance of the genetic algorithm [22]. Generally, the population size depends on the length of the chromosome. The population size should be at least equal to the length of the chromosome. Here, the population size is taken to be 2.5 times the length of the chromosome [5].

\subsubsection{Evaluation Function}

Before performing the crossover function to produce offspring, each solution in the population pool should be evaluated with respect to its design objective to determine its fitness value. Here, the design objective is the minimization of the heterogeneity. Genetic algorithm works with maximization function. Hence, the objective function is transformed into maximization using the following transformation.

$$
F_{i}=1 /\left(1+T_{i}\right)
$$

where, $T_{i}$ is the value of objective function of the chromosome $i$ of the population and $F_{i}$ is the value of the fitness function of the chromosome $i$.

\subsubsection{Genetic Operators}

To create the next generation, a new set of chromosomes called off springs are formed by the execution of genetic operators such as reproduction, crossover and mutation. The purpose of the reproduction operator is to select parents for the next generation of solutions. Parents are selected according to their fitness values. Stochastic sampling without replacement policy $[4,5]$ is used in the genetic algorithm for reproduction. The crossover operator produces children by exchanging information contained in the parents. The crossover operator creates new potential solutions (children) by exchanging portions of the parent solutions and in this work two-point crossover is used. Mutation performs a secondary role in the operation of genetic algorithms. Mutation is needed because even though reproduction and crossover effectively search and recombine chromosomes, occasionally they may lose some potentially useful genetic material (decision nodes at certain places which under certain conditions may improve the overall objective function value). The mutation is carried out with a very small probability called probability of mutation.

After, the genetic operators have been applied, new strings are created and these strings are called children or offsprings. The poorly performing offsprings are replaced in the new generation with a replacement strategy. The offsprings are evaluated with respect to the evaluation function. The goal of the replacement strategy is to create generations of solutions that, on an average, outperform the previous generation. This is achieved by restricting entry to the new population to only those children that are better than members of the existing population. This can be accomplished by comparing the fitness values of the chromosomes of the old population with the chromosome of new generation. The fittest among the two will turn out to be the old population for the next generation. Some times less fit children are also allowed to continue to exist and become part of the next generation for the purpose of guiding the algorithm to come out of the local optima.

The termination criteria: if there is no improvement in the best solution found for a pre-specified number of generations the algorithm terminates or if the algorithm reaches a maximum number of generations the algorithm terminates. This algorithm is coded in MATLAB.

The value for certain parameters of the GA are as follows:

- $\quad$ Probability of crossover $=0.85$

- $\quad$ Probability of mutation $=0.1$

- $\quad$ Pre-specified number of generations $=100$

- $\quad$ Maximum generations $=1000$

\subsection{Part assignment heuristic}

Part assignment to cells is carried out for the solution obtained using the GA. A part is assigned to a cell in which it is having the maximum number of operations to get a good block diagonalisation. 


\subsection{Performance measure}

The grouping efficacy proposed by Kumar and Chandrasekharan [23] is the most widely used and popular measure for checking the quality of solutions and we also used the same for finding the goodness of the solutions.

The grouping efficacy, GE, can be defined as

$$
G E=\frac{(m-e)}{(m+v)}
$$

Where,

\section{$G E$ - Grouping Efficacy}

$m$ - Total number of $1 \mathrm{~s}$ in matrix A;

$e$ - Total number of 1 s (exceptional

elements) outside the diagonal blocks;

$v$ - Total number of 0s (voids) inside the diagonal blocks.

\subsection{Algorithm}

The stepwise methodology of the whole algorithm is given below.

Step 1: Input the part-machine matrix, the genetic algorithm parameters to the model, minimum number of cells and maximum number of cells.

Step 2: Set number of cells equal to minimum number of cells

Step 3: Initialize the population based on the number of cells.

Step 4: Calculate heterogeneity for each member of the population and convert it into corresponding fitness value.

Step 5: Reproduce strings using remainder stochastic sampling without replacement policy.

Step 6: Strings are crossed using two-point crossover operator.

Step 7: Mutation is performed.

Step 8: Carryout the replacement strategy and evaluate the strings.

Step 9: Go to step 10 if the termination criteria is met, else go to step 5.

Step 10: Select the best configuration.

Step 11: Assign pats to the cells based the part assignment heuristic for the selected configuration.
Step 12: Calculate grouping efficacy value for the selected configuration.

Step 13: Increment number of cells by one and go to step 3 if maximum number of cells is not reached, else go to next step.

Step 14: Select the configuration with maximum grouping efficacy.

\section{NUMERICAL ILLUSTRATION OF THE MODEL}

The proposed model is tested on 7 problems from literature to analyze its efficiency. The selected problems ranges from 5 machines $\square \times 7$ parts to 24 machines $\times 40$ parts, and includes well-structured as well as ill-structured matrices. We compare the grouping efficacy obtained for the seven problems solved using our model with the grouping efficacies using the following seven approaches to illustrate the efficiency of our model:

ZODIAC (Chandrasekharan \& Rajagopalan, 1987) [12]

GRAFICS (Srinivasan \& Narendran, 1991) [11]

GATSP — Genetic algorithm (Cheng et al., 1998) [24]

GP - Genetic programming (Dimopoulos \& Mort, 2001) [25]

EA - Evolutionary algorithm (Jose and Mourisio, 2004 ) [2]

TPA - Two phase algorithm (Doulabi et al., 2009) [26]

The proposed algorithm is applied to seven problems given in Table 1. This table shows the matrix sizes, their sources, and the grouping efficacy values from various approaches and the proposed model. Also, this table shows that the proposed model provides better results for the two problems and for the other problems the performance of the proposed model is same as the best available in literature. So, the computational results show the efficiency of the proposed model in the design of manufacturing cell. Table 2 is the input matrix and Table 3 is the output for the problem number two where an improved solution is obtained when the proposed model is applied. This is an indication of the power of heterogeneity concept in designing CMS

Table 1: Grouping Efficacy

\begin{tabular}{|c|c|c|c|c|c|c|c|c|c|c|}
\hline Problem & \multicolumn{2}{|l|}{ Source of the problem } & $\begin{array}{c}\text { Size } \\
(\mathbf{M} \times \mathbf{P})\end{array}$ & ZODIAC & GRAPHICS & GATSP & GP & EA & TPA & $\begin{array}{c}\text { PROPOSED } \\
\text { MODEL }\end{array}$ \\
\hline 1 & \multicolumn{2}{|l|}{ King and Nakornchai (1982) } & $5 * 7$ & 73.68 & 73.68 & - & - & 73.68 & 73.68 & 73.68 \\
\hline 2 & \multicolumn{2}{|l|}{ Kusiak and Chow (1987) } & $7 * 11$ & 39.13 & 53.12 & 46.88 & - & 53.13 & - & 58.62 \\
\hline 3 & \multicolumn{2}{|l|}{ Chan and Milner (1982) } & $10 * 15$ & 92.00 & 92.00 & 92.00 & & 92.00 & 92.00 & 92.00 \\
\hline 4 & \multicolumn{2}{|l|}{ Srinivasan and Narendran (1990) } & $16 * 30$ & 67.83 & 67.83 & - & - & 67.83 & 67.83 & 68.31 \\
\hline 5 & \multirow{3}{*}{$\begin{array}{c}\text { Chandrasekharan and Rajagopalan } \\
(1989)\end{array}$} & $\mathrm{a}$ & $24 * 40$ & 100 & 100 & 100 & 100 & 100 & 100 & 100 \\
\hline 6 & & $\mathrm{~b}$ & $24 * 40$ & 85.11 & 85.11 & 85.11 & - & 85.11 & 85.11 & 85.11 \\
\hline 7 & & $\mathrm{c}$ & $24 * 40$ & 73.51 & 73.51 & 73.03 & 73.51 & 73.51 & 73.51 & 73.51 \\
\hline
\end{tabular}

Table 2: Part-machine incident matrix for problem 2

\begin{tabular}{|c|c|c|c|c|c|c|c|c|c|c|c|c|}
\hline & \multicolumn{12}{|c|}{ Parts } \\
\hline \multirow{8}{*}{ 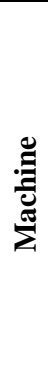 } & & 1 & 2 & 3 & 4 & 5 & 6 & 7 & 8 & 9 & 10 & 11 \\
\hline & 1 & 0 & 1 & 1 & 0 & 0 & 0 & 1 & 0 & 0 & 0 & 0 \\
\hline & 2 & 1 & 0 & 0 & 0 & 1 & 0 & 0 & 0 & 0 & 0 & 1 \\
\hline & 3 & 0 & 0 & 0 & 0 & 0 & 0 & 0 & 0 & 0 & 1 & 1 \\
\hline & 4 & 1 & 0 & 1 & 0 & 0 & 1 & 0 & 0 & 0 & 0 & 0 \\
\hline & 5 & 0 & 0 & 0 & 0 & 1 & 0 & 0 & 1 & 0 & 0 & 0 \\
\hline & 6 & 1 & 0 & 0 & 1 & 0 & 0 & 0 & 1 & 1 & 1 & 0 \\
\hline & 7 & 0 & 0 & 1 & 1 & 0 & 1 & 1 & 0 & 1 & 0 & 0 \\
\hline
\end{tabular}

Table 3: Block diagonal structure for the problem number 2 obtained using the proposed model

\begin{tabular}{|c|c|c|c|c|c|c|c|c|c|c|c|c|}
\hline & \multicolumn{12}{|c|}{ Parts } \\
\hline \multirow{8}{*}{ 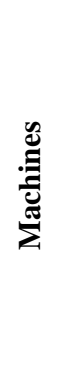 } & & 2 & 7 & 5 & 8 & 10 & 11 & 1 & 3 & 4 & 6 & 9 \\
\hline & 1 & 1 & 1 & 0 & 0 & 0 & 0 & 0 & 1 & 0 & 0 & 0 \\
\hline & 2 & 0 & 0 & 1 & 0 & 0 & 1 & 1 & 0 & 0 & 0 & 0 \\
\hline & 5 & 0 & 0 & 1 & 1 & 0 & 0 & 0 & 0 & 0 & 0 & 0 \\
\hline & 3 & 0 & 0 & 0 & 0 & 1 & 1 & 0 & 0 & 0 & 0 & 0 \\
\hline & 4 & 0 & 0 & 0 & 0 & 0 & 0 & 1 & 1 & 1 & 0 & 0 \\
\hline & 6 & 0 & 0 & 0 & 1 & 1 & 0 & 1 & 0 & 0 & 1 & 1 \\
\hline & 7 & 0 & 1 & 0 & 0 & 0 & 0 & 0 & 1 & 1 & 1 & 1 \\
\hline
\end{tabular}




\section{CONCLUSION}

Wide range of methods based on array manipulation, hierarchical and non-hierarchical clustering, neural-network based approach, mathematical programming and algorithms, etc are generally used in CMS design. Most of the techniques use intercell movements as an objective to be minimised. This paper introduces a concept of heterogeneity of CMS instead of intercell movements and it is used in the proposed mathematical model. The proposed non-linear integer programming model is suitable for machine cell formation which uses the part-machine incidence matrix. A genetic algorithm based solution procedure is developed for the model and coded in MATLAB. Seven problems from the literature are solved using the model and the results are compared with previously available models and algorithms with respect to the grouping efficacy. The results show that better configurations are obtained using the proposed model and the heterogeneity can be considered as an objective for cell design.

\section{REFERENCES}

[1] King, J. R., 1980. Machine-component grouping in production flow analysis; an approach using rank order clustering algorithm. International Journal of Production Research, 18, 2, 213-232.

[2] Goncalves, J. F. and Resende, M.G.C., 2004. An evolutionary algorithm for manufacturing cell formation. Computers \& Industrial Engineering, 47, 247-273.

[3] Nair G. J. and Narendran T. T., 1998. CASE: A clustering algorithm for cell formation with sequence data; International Journal of Production Research, 36, 157179.

[4] Wicks, E. M. and Reasor, R. J., 1999. Designing cellular manufacturing systems with dynamic part Populations. IIE Transactions, 31, 11-20.

[5] Pillai, V.M. and Subbarao, K.A., 2008. Robust cellular manufacturing system design for dynamic part population using a genetic algorithm. International Journal of Production Research, 46 ,1, 5191 -5210.

[6] Defersha, F. M. and Chen, M., 2008. A parallel genetic algorithm for dynamic cell formation in cellular manufacturing systems. International Journal of Production Research, 46,22, 6389-6413.

[7] Shang, J. S. and Tadikamalla, P. R., 1998. Multicriteria design and control of a cellular manufacturing system through simulation and optimization. International Journal of Production Research, 36 ,6, 1515-1528.

[8] McCormick, W. T. Scweitzer, P. J. and White, T. W., 1972. Problem decomposition and data reorganization by a cluster technique. Operations Research, 20, 993-1009.

[9] McAuley, J., 1972. Machine grouping for efficient production. Production Engineer, 51, 2,.53-57.

[10] Seifoddini, H. \& Wolfe, P. M., 1986. Application of the similarity coefficient method in group technology. IIE Transactions, 18, 3, 266-270.

[11] Srinivasan, G., and Narendran, T. T, 1991. GRAFICSA nonhierarchical clustering-algorithm for group technology. International Journal of Production Research, 29, 3, 463-478.

[12] Chandrasekharan, M. P. and Rajagopalan, R., 1987. ZODIAC - An algorithm for concurrent formation of part families and machine cells. International Journal of Production Research, 25, 6, 835-850.
[13] Askin, R. G. and Chiu, K. S., 1990. A graph partitioning procedure for machine assignment and cell formation in group technology. International Journal of Production Research, 28, 555-1572.

[14] Choobineh, F., 1988. A framework for the design of cellular manufacturing systems. International Journal of Production Research, 26(7), 1161-1172.

[15] Srinivasan, G., Narendran, T. T. and Mahadevan, B., 1990. An assignment model for the part-families problem in group technology. International Journal of Production Research, 28, 1, 145-152.

[16] Adil, G. K., Rajamani, D. and Strong, D., 1997. Assignment allocation and simulated annealing algorithms for cell formation. IIE Transactions, 29, 5367.

[17] Yasuda, K., Hu, L. and Yin, Y., 2005. A grouping genetic algorithm for the multi-objective cell formation problem. International Journal of Production Research, $43,4,829-853$

[18] Adenso-Diaz, B. and Lozano, S., 2008. A model for the design of dedicated manufacturing cells. International Journal of Production Research, 46 ,2, 301-319.

[19] Chen, C. L, Cotruvo, N. A. and Baek,W., 1995. A simulated annealing solution to the cell formation problem. International Journal of Production Research, 33, 2601-2614.

[20] Singh, N. and Rajamani, D., 1996 Cellular Manufacturing Systems Design, Planning and Control, Chapman \& Hall: London, UK

[21] Ephsibah, E. P., 2010, Cost Effective Approach on Feature Selection using Genetic Algorithms and LSSVM Classifier, International journal of computer applications, IJCA Special Issue on "Evolutionary Computation for Optimization Techniques"

[22] Gupta, R. M. and Tompkins, J. A., 1982. An examination of the dynamic behavior of part families in group technology, International Journal of Production Research, 20, 73-86.

[23] Kumar, K. R., \& Chandrasekharan, M. P.,1990. Grouping efficacy: A quantitative criterion for goodness of block diagonalforms of binary matrices in group technology. International Journal of Production Research, 28, 2, 233-243.

[24] Cheng, C. H., Gupta, Y. P., Lee, W. H., \& Wong, K. F., 1998. A TSP-based heuristic for forming machine groups and part families. International Journal of Production Research, 36, 5, 1325-1337.

[25] Dimopoulos, C., \& Mort, N., 2001. A hierarchical clustering methodology based on genetic programming for the solution of simple cell-formation problems. International Journal of Production Research, 39, 1, 119.

[26] Dolabi, S. H. H., Hojabri, H., Alagheband, S. A. S., Jaafari, A. A. and Davoudpour, H., 2009. Two phase approach for solving cell formation problem in cellular manufacturing, Proceedings of the World Congress on Engineering and Computer Science, October 20-22, 2009, San Francisco, USA. 